\title{
Linx
}

Revue des linguistes de l'université Paris X Nanterre

$80 \mid 2020$

L'héritage de Jean Dubois et Françoise Dubois-Charlier

\section{Un problème de syntaxe : la subordination en when / quand, dite "subordination inverse"}

A syntactic and enunciative analysis of so-called 'reverse subordination' in temporal subclauses in French and English

Jean-Marie Merle

\section{CpenEdition}

Journals

Édition électronique

URL : http://journals.openedition.org/linx/6397

DOI : 10.4000/linx.6397

ISSN : 2118-9692

Éditeur

Presses universitaires de Paris Nanterre

Référence électronique

Jean-Marie Merle, «Un problème de syntaxe : la subordination en when / quand, dite « subordination inverse » », Linx [En ligne], 80 | 2020, mis en ligne le 10 juillet 2020, consulté le 05 août 2020. URL http://journals.openedition.org/linx/6397 ; DOI : https://doi.org/10.4000/linx.6397

Ce document a été généré automatiquement le 5 août 2020

Département de Sciences du langage, Université Paris Ouest 


\title{
Un problème de syntaxe : la
} subordination en when / quand, dite «subordination inverse »

\author{
A syntactic and enunciative analysis of so-called 'reverse subordination' in \\ temporal subclauses in French and English
}

Jean-Marie Merle

\section{Introduction}

1 Jean Dubois et Françoise Dubois-Charlier ont toujours donné une place centrale à la syntaxe dans la construction du sens.

2 Cet article ${ }^{1}$ a pour but, en hommage à Jean Dubois et à Françoise Dubois-Charlier, de revenir sur un phénomène syntaxique, en anglais et en français, que l'on a souvent la tentation de définir à partir de gloses - la subordination temporelle dite « subordination inverse » :

Il avait fini son travail sur les ruches [...] quand il aperçut David Kellerman qui s'activait (Teissier)

He was just congratulating himself on his keen sense of observation and talking out what he would say to the police in his mind, when the truck smashed into his car [...] (Bradbury).

Les gloses auxquelles on a recours remanient la syntaxe (Alors qu'il avait fini son travail [...] il aperçut David Kellerman / As he was congratulating himself [...] the truck smashed into his car).

Or les gloses ont pour fonction de fournir des approximations de sens, et elles peuvent reformuler la syntaxe à l'infini. L'observation montrera que la construction du sens s'appuie bien en l'occurrence sur une syntaxe de subordination, mais qu'on n'a pas affaire à une « subordination inverse $»^{2}$. 
On commencera par une présentation du problème ( $(1)$. Puis on passera par trois étapes successives. Comme le phénomène de "subordination inverse " concerne les repérages temporels, la première étape $(\$ 2)$ sera un retour sur le repérage temporel apporté par les subordonnées intégratives ${ }^{3}$ adverbiales. La deuxième étape $(\S 3)$ reviendra sur les caractéristiques des relatives à antécédent non nominal. La troisième étape (\$ 4) sera consacrée aux relatives continuatives. Le $\$ 5$ sera consacré à la synthèse des observations précédentes et aux conclusions : cette subordination temporelle n'est pas «subordination inverse » et, comme le fait toujours la syntaxe, elle apporte sa contribution à la cohérence discursive et à la construction du sens.

\section{La « subordination inverse » en français et en anglais}

Riegel, Pellat \& Rioul (1998: 507) présentent «l'effet de subordination inverse» temporelle en ces termes :

Citation 1. La temporelle postposée peut [...] se prêter à l'effet de subordination inverse pour peu que la principale soit à un temps de l'inaccompli, par exemple l'imparfait: Trois heures sonnaient au clocher du village quand le drame se produisit. La proposition qui est formellement la principale présente en réalité le thème de la phrase et représente les circonstances (elle en indique le cas échéant le repérage temporel, par exemple la datation), c'est la subordonnée qui en est le propos et qui exprime le fait essentiel. (Riegel, Pellat \& Rioul, 1998 : 507)

\section{Trois remarques}

7 Remarque 1 - L'idée de «subordination inverse » entre en contradiction avec les faits syntaxiques et avec les faits discursifs. L'idée de « fait principal », quant à elle, contient une part d'arbitraire, et par ailleurs la subordination est compatible avec le «fait principal ». Il est fréquent que la subordonnée contienne le «fait principal», comme dans « Meurtre d'une journaliste à Malte: le Premier ministre annonce qu'il démissionnera en janvier » (titre du Dauphiné Libéré, 2019) : le fait principal n'est pas l'existence d'une annonce (Le premier ministre fait une annonce), mais le contenu de l'annonce (Le premier ministre démissionnera en janvier). La complétive n'en est pas pour autant principale, et on n'a pas affaire à une subordination "inverse ", mais à la mise en place d'une situation d'énonciation dérivée dans la principale enchâssante, et d'un contenu de discours rapporté dans la subordonnée enchâssée.

Remarque 2 - Dans l'exemple donné par Riegel, Pellat \& Rioul, le «temps de l'inaccompli » choisi par les auteurs et qui permet «l'effet de subordination inverse » est l'imparfait (sonnaient). Outre le «temps de l'inaccompli», on peut considérer que c'est le caractère statique du contenu de la principale qui permet cet effet. Tout autant qu'avec l'imparfait, « l'effet de subordination inverse » est en effet compatible avec un plus-que-parfait - qui marque « l'aspect accompli $»^{4}$ (Riegel, Pellat \& Rioul, 1998 : 310) dans la principale, comme dans l'exemple suivant, où les deux, plus-que-parfait (avait fini) et imparfait (se trouvait), coexistent dans les deux principales coordonnées entre elles :

(1) Il avait fini son travail sur les ruches et il se trouvait à une cinquantaine de mètres du ruisseau quand il aperçut David Kellerman qui s'activait, à moitié caché par les pommiers. (Teissier, 2013) 
La saisie aspectuelle, qu'elle soit sécante (inaccompli) ou transcendante (accompli), a pour effet de figer le dynamisme d'un procès. C'est pour cette raison que ces deux types de saisies sont à même de représenter un état de fait préconstruit susceptible de fournir un repère temporel à une subordonnée continuative dans les structures examinées ici.

10 Remarque 3 - Les "circonstances » dont parlent Riegel, Pellat \& Rioul sont des circonstances sémantiques. Benzitoun $(2013: 423)$ fait à juste titre la remarque suivante à propos de la citation 1 :

Citation 2. Comme l'exprime bien la citation [citation 1 de Riegel, Pellat \& Rioul], elle [elle = l'idée de « subordination inverse »] est parfois le résultat d'un mélange des niveaux d'analyse - visiblement entre syntaxe et sémantique. (Benzitoun, 2013 : 423)

11 On trouve la même idée, mais exprimée en termes normatifs, chez Miller \& Weinert (citation 3, 1998 : 95) à propos de l'exemple 2 :

(2) She shone the light along the dark passage when suddenly she saw a big rat. (Miller \& Weinert, 1998: 95)

Citation 3. The when clause is not a subordinate clause but a main clause and when can be treated as a conjunction joining two main clauses - a very common construction in spontaneous spoken English, but not one that is in the canon of formal written English. (Miller \& Weinert, 1998: 95)

\section{Autres remarques}

12 She shone the light along the dark passage est susceptible de deux interprétations : l'une aoristique, représentation d'un événement borné (elle braqua la lumière sur le passage obscur quand tout à coup elle aperçut un gros rat); l'autre atélique, représentation d'un état de fait non borné (elle braquait la lumière sur le passage obscur quand tout à coup elle aperçut un rat).

13 1. La première est celle que rejettent Miller \& Weinert, mais qui peut éventuellement s'interpréter comme un enchaînement événementiel de l'ordre de she suddenly saw a rat and she shone the light along the dark passage ( $\mathrm{p} 2$ déclenche $\mathrm{p} 1^{5}$ ).

14 2. La seconde correspond à la structure qui nous intéresse, mais cette seconde lecture ne fait pas de la subordonnée une principale, ni de la principale une subordonnée.

Dans ce type de construction, l'aspect be + Ving garantit la sélection d'un état de fait dans la principale, comme dans les exemples 3 et 4 , mais, d'une manière générale, même en l'absence de be + Ving, on a toujours affaire à un état de fait - à un contenu propositionnel stabilisé, préconstruit par rapport à $\mathrm{p} 2$ - comme dans l'interprétation 2 de l'exemple 2, ci-dessus, ou comme dans les trois exemples suivants $(3,4$ et 5$)$ :

(3a) He was turning drearily away, when he saw a drop of blood fall from the averted wound into the baby's fragile, glistening hair. (D.H. Lawrence)

(4a) He was just congratulating himself on his keen sense of observation and talking out what he would say to the police in his mind, when the truck smashed into his car. (Ray Bradbury)

(5a) Mr. Pepper looked up sharply, and was about to put a question when Willoughby continued: [...] (V. Woolf, Jacob's Room)

L'idée d'une « subordination inverse » est inspirée par une glose du type :

(3b) As he turned drearily away, he saw a drop of blood.

(4b) As he was congratulating himself, the truck smashed into his car.

(5b) As he was about to put a question, Willoughby continued [...]. 

apporté par les subordonnées intégratives adverbiales traditionnellement reconnues comme subordonnées; 2/ les relatives à antécédent non nominal ; 3/ les relatives continuatives à antécédent nominal.

En conclusion, on verra que ces subordonnées temporelles dites à «effet de subordination inverse » sont en fait des subordonnées continuatives dont l'antécédent est la coordonnée temporelle de la principale, représentée à l'intérieur de la subordonnée par l'adverbe relatif when.

\section{Etape 1 - Le repérage temporel apporté par les subordonnées intégratives adverbiales}

Soient les trois exemples suivants :

(6) 'They jumped apart when I opened the door.' (M. Spark)

(7) 'Were you inside or outside the room when you saw them?' (M. Spark)

(8) Then when the calculation was finished she said, '[...]' (M. Spark)

Dans l'exemple 6, on a affaire à deux événements ( $\mathrm{p} 1$ they jumped apart / $\mathrm{p} 2$ I opened the door).

Dans l'exemple 7 on a affaire à un état de fait et à un événement (p1 were you inside or outside the room? / p2 you saw them).

Dans l'exemple 8 - comme dans l'exemple 7 -, à un état de fait et à un événement (p2 the calculation was finished / 1 she said [...]), mais dont la répartition entre subordonnée p2 et principale p1 est l'inverse de celle de l'exemple 7.

Un événement ou un état de fait possède intrinsèquement des coordonnées, et notamment une coordonnée temporelle. C'est ce qui permet d'exploiter un contenu propositionnel renvoyant à un état de fait ou à un événement comme repère temporel d'un autre contenu propositionnel. Dans ces trois exemples, c'est la proposition en when (when I opened the door / when you saw them / when the calculation was finished) qui est exploitée comme repère temporel de la principale (respectivement, de they jumped apart / were you inside or outside the room? / she said [...]).

Le rôle de when, qui établit une relation qualitative avec le paradigme du temps ${ }^{6}$, est :

1/ de matérialiser sans spécification (propriété du submorphème wh-) la coordonnée temporelle (propriété du submorphème -en) de la subordonnée : when est adverbe de temps et a une fonction adverbiale à l'intérieur de chaque subordonnée; when I opened the door / when you saw them / when the calculation was finished = (at) the moment when ${ }^{7} /$ the moment that I opened the door / etc.;

2/ syntaxiquement, de combiner sa fonction adverbiale avec une fonction de subordonnant ;

3 / de donner à la subordonnée un statut de repère temporel pour la principale : autrement dit, la subordonnée adverbiale a la même fonction par rapport à la principale que when par rapport à la subordonnée. ${ }^{8}$

Première glose (approximation de sens) de when dans $\mathrm{p} 2$ :

At some point in time I opened the door / At some point in time you saw them / At some point in time the calculation was finished. Cette glose rend compte $d u$ caractère non quelconque et, à ce stade, non spécifié (some / wh-) du repère temporel (point in time / -en). 
L'articulation opérée par when dans la relation de subordination a pour effet que la coordonnée temporelle de $\mathrm{p} 2$ sert également de coordonnée temporelle de p1. C'est de là que vient cette fonction articulatoire de when, que l'on peut gloser ainsi :

At the same point in time they jumped apart / At that point in time were you inside or outside the room? / At that point in time she said [...]. A ce stade, le repère temporel est spécifié par $\mathrm{p} 2$ (changement de détermination dans la glose : at some point in time $\Rightarrow>$ at the same / that point in time).

C'est ainsi qu'un état de fait (the calculation was finished dans l'exemple 8) ou un événement (I opened the door dans l'exemple 6, you saw them dans l'exemple 7) peut servir de repère temporel à un événement (she said [...], dans l'exemple 8 / they jumped apart, dans l'exemple 6) ou à un état de fait (were you inside or outside the room? dans l'exemple 7). Comme on l'a vu, le subordonnant when à l'intérieur de la subordonnée, et la proposition subordonnée elle-même ('when p2') par rapport à la principale, ont une fonction syntaxique adverbiale d'adjoint et ils font partie des circonstances naturelles (sémantico-référentielles) de tout état de fait ou de tout événement.

\section{Etape 2 - Relatives à antécédent non nominal}

Soient les trois exemples suivants :

(9) He said he was arrogant, which I don't think he is. (Huddleston \& Pullum 2002)

(10) He set out to redeem himself, which he eventually did. (H \& P 2002)

(11) He wouldn't let us defend ourselves, which was completely unfair. (H \& P 2002)

Les relatives à antécédent non nominal sont appositives, c'est-à-dire co-prédicatives9 Une glose (approximation de sens ${ }^{10}$ ) peut se faire à l'aide d'une coordination :

(9') He said he was arrogant but I don't think he is.

(10') He set out to redeem himself, and he eventually did so.

(11') He wouldn't let us defend ourselves, and / but this was completely unfair.

31 Ces gloses $\left(9^{\prime}\right.$ à $\left.11^{\prime}\right)$ sont des énoncés bi-prédicatifs: les principales p1 et les subordonnées p2 des exemples 9 à 11 sont chacune déterminées par sa ou ses modalités. On remarque cependant que c'est le jugement modal apporté par p2 (la relative appositive) qui motive cette structuration, que ce jugement modal concerne une partie de p1 (exemples 9 et 10) ou la totalité de p1 (la principale, dans l'exemple $11)^{11}$, et que la modalité de la relative p2 est le plus souvent de l'ordre de la plurimodalité12 : assertion d'un jugement appréciatif (et éventuellement incertitude épistémique) dans l'exemple 9 ; assertion de validation dans l'exemple 10 ; assertion d'un jugement appréciatif dans l'exemple 11.

L'antécédent est non nominal dans les trois cas :

33 - dans l'exemple 9, la proforme ${ }^{13}$ complexe disjointe which...be 㕐 be which articule la subordonnée $\mathrm{p} 2$ sur la propriété (le prédicat) be arrogant ;

34 - dans l'exemple 10, la proforme complexe which... did 胳 did which articule la subordonnée sur le prédicat redeem himself;

35 - dans l'exemple 11, le relatif which articule la subordonnée sur tout le contenu propositionnel de $\mathrm{p} 1$. 

il s'agit de la présélection d'un procès dans l'exemple 9 et dans l'exemple $10^{14}$, d'un état de fait (he wouldn't let us defend ourselves) dans l'exemple 11.

\section{Etape 3 - Les relatives continuatives}

Soient les exemples suivants :

(12) Vince slammed the door, which oliver reopened immediately. (exempletype)

(13) This was not dramatically satisfying to Sandy who went out of the classroom. (M. Spark)

(14) The Brodie set decided to keep the incident to themselves lest, if it should spread to the rest of the class, it should spread wider still and eventually to someone's ears who would get Monica Douglas into trouble. (Muriel Spark, The Prime of Miss Jean Brodie)

(15) 'Hello, Cinderella, I am your fairy godperson, or individual deity proxy, if you prefer. So, you want to go to the ball, eh? And bind yourself into the male concept of beauty? Squeeze into some tight-fitting dress that will cut off your circulation? Jam your feet into high-heeled shoes that will ruin your bone structure? Paint your face with chemicals and make-up that have been tested on nonhuman animals?' (James Finn Garner, 'Cinderella', Politically Correct Bedtime Stories)

Dans l'exemple 12, la relative which Oliver reopened immediately s'articule via which sur son antécédent dans $\mathrm{p} 1$ (the door). Which articule ainsi $\mathrm{p} 2$ sur $\mathrm{p} 1$ dans une relation appositive (de co-prédication), qui partage des propriétés avec les exemples 9-11 de la section précédente ( $\$ 3)$, notamment la possibilité de gloser à l'aide d'une coordination (Vince slammed the door and / but Oliver reopened it immediately). Mais on observe aussi des divergences: 1/ l'antécédent de which est nominal (the door); 2/ p2 représente un développement qui fait suite à l'événement ou à l'état de fait représenté par p1. D'où le terme de relative appositive continuative.

Dans l'exemple 13, on a affaire à un phénomène analogue: this was not dramatically satisfying to Sandy, and she went out of the classroom serait une glose possible. Dans la glose de l'exemple 12, on peut étoffer la coordination à l'aide de then (and then), alors que dans la glose de l'exemple 13, l'étoffement se ferait à l'aide de so (and so). Pour autant, la conséquence dite dans $\mathrm{p} 2$ entre toujours dans une relation séquentielle avec $\mathrm{p} 1$.

Dans l'exemple 14, la même glose par une coordination est possible et l'interprétation est toujours séquentielle. En réduisant l'énoncé : the rumour might spread to someone's ear and that person might get Monica into trouble. On remarque que c'est who (référence qualitative au paradigme de la personne) qui sélectionne l'articulation de la continuative sur someone et non sur someone's ear. C'est cette sélection qui permet de construire l'interprétation continuative.

Dans l'exemple 15, on n'a pas affaire à des relatives appositives, mais à deux relatives intégrées (épithétiques) : that will cut off your circulation est intégrée dans le syntagme some tight-fitting dress that will cut off your circulation; that will ruin your bone structure est intégrée dans le syntagme high-heeled shoes that will ruin your bone structure. Les relatives intégrées, comme on le sait, sont épithétiques et, comme toutes les formes ${ }^{15}$ à fonction épithétique, elles représentent une caractéristique - propriété saillante de l'antécédent qu'elles caractérisent -, une propriété pertinente et saillante, qui peut être appréciative ${ }^{16}$ et qui peut éventuellement ${ }^{17}$ avoir une exploitation référentielle (soit 
restrictive, soit déterminative). Cette propriété saillante peut également être continuative, comme ici dans ces deux occurrences, ce qui signifie que la caractéristique donnée dans la relative $\mathrm{p} 2$, that will cut off your circulation / that will ruin your bone structure, est issue de la validation de $\mathrm{p} 1$ (<you-squeeze into some tight-fitting dress> ; <you-jam your feet into high-heeled shoes>). La glose est possible à l'aide d'un coordonnant (you squeeze into some tight-fitting dress and it will cut off your circulation; you jam your feet into high-heeled shoes and they will ruin your bone structure), ou à l'aide de l'empilement protase-apodose d'un système hypothétique potentiel (if you squeeze into some tight-fitting dress, it will cut off your circulation; if you jam your feet into high-heeled shoes, they will ruin your bone structure) et la validation de $\mathrm{p} 2$ (le contenu de la relative) dépend et découle de la validation de 1 (le contenu de la principale). On a donc affaire à un cas de relative intégrée, mais continuative.

\section{Synthèse}

Soient, encore, les exemples suivants:

(3a) He was turning drearily away, when he saw a drop of blood fall from the averted wound into the baby's fragile, glistening hair. (D.H. Lawrence)

(4a) He was just congratulating himself on his keen sense of observation and talking out what he would say to the police in his mind, when the truck smashed into his car. (Ray Bradbury)

(5a) Mr. Pepper looked up sharply, and was about to put a question when Willoughby continued: [...] (V. Woolf, Jacob's Room)

L'hypothèse faite ici, étayée par l'observation et l'analyse syntaxique, est que l'on a affaire à une relative appositive, continuative, à antécédent temporel. Une approximation de sens intégrant à la fois les propriétés syntaxiques et discursives de cette structure pourrait être :

(3c) He was turning drearily away, at which point (in time) he saw a drop of blood $[\ldots]$

(4c) he was congratulating himself, at which point the truck smashed into his car [...]

(5c) Mr. Pepper looked up sharply, and was about to put a question, at which point in time Willoughby continued: [...]

Ce que ces subordonnées ont en commun avec les subordonnées temporelles des exemples de la série 6-8 (§ 2):

(6) 'They jumped apart when I opened the door.' (M. Spark)

(7) 'Were you inside or outside the room when you saw them?' (M. Spark)

(8) Then when the calculation was finished she said, '[...]' (M. Spark)

Toutes ces subordonnées ont pour subordonnant l'adverbe when, qui a une fonction de circonstant à l'intérieur de la subordonnée aussi bien dans les exemples 3-5 que dans les exemples 6-8.

Voici les divergences :

- Les subordonnées temporelles when I opened the door / when you saw them / when the calculation was finished des exemples 6-8 sont des intégratives adverbiales : l'adverbe when n'a pas d'antécédent; ces subordonnées servent de repère temporel aux principales they jumped apart / were you inside or outside the room? / she said, [...]. C'est la coordonnée temporelle de la subordonnée p2 qui est exploitée comme repère de la principale $\mathrm{p} 1$. 
Les subordonnées temporelles when he saw a drop of blood / when the truck smashed into his car / when Willoughby continued des exemples 3-5 ne sont pas des repères temporels : elles sont repérées par rapport aux principales he was turning drearily away / He was congratulating himself / Mr Pepper [...] was about to put a question ; la relation de repérage s'appuie sur le caractère statique et préconstruit du contenu propositionnel des principales (cf. was turning / was congratulating himself / was about to put a question); la relation de repérage s'opère via la relation entre when et son antécédent, qui est la coordonnée temporelle de chaque principale ; c'est pour cette raison que l'ordre est contraint dans les constructions qui nous intéressent, alors qu'il ne l'est pas dans le cas des intégratives adverbiales des exemples 6-8; la coordonnée temporelle de la principale n'est pas spécifiée, mais il est inutile qu'elle le soit : comme elle est inhérente à tout état de fait (et à tout événement) et toujours disponible, elle est toujours exploitable ; ce qui active son exploitation, c'est précisément l'emploi de when : le choix du relatif oriente vers l'identification implicite d'un antécédent ( $c f$. le phénomène analogue observé dans l'exemple 14 , au $\S 4$, où l'antécédent est sélectionné par le relatif who lui-même).

Les subordonnées temporelles dites " à effet de subordination inverse " sont en fait des continuatives, comme dans les exemples de la série 12-14 ( $\$ 4)$, dont l'antécédent n'est pas nominal, comme dans les exemples de la série 9-11 (\$3). En l'occurrence, comme on vient de le rappeler, l'antécédent non nominal est la coordonnée temporelle de la principale $p 1$, la sélection du paramètre temporel étant activée et annoncée par when. Sa pertinence tient à ce que p1 est un contenu propositionnel donné comme préconstruit et stabilisé (he was turning drearily away / He was congratulating himself / Mr Pepper was about to put a question), deux caractéristiques qui s'accordent parfaitement avec le statut de repère de $p 1$.

On a donc affaire à des relatives appositives continuatives à antécédent temporel, selon le modèle : Etat de fait p1, at which point in time / when événement $\mathrm{p} 2$. Le principe des continuatives est d'articuler sur la principale un développement qui en est le prolongement. C'est ce qui justifie la subordination, et c'est ce qui justifie l'ordre dans lequel sont placées les deux propositions. Le développement apporté par les continuatives est de l'ordre de l'enchaînement propre au récit : Declerck 1997 en arrive à des conclusions analogues et les nomme narratives.

On retrouve ce même type de subordination dans l'exemple 16 :

(16) Hardly had she announced this fell decision when she reversed it. (E.M. Forster, A Room with a View)

(16') She (had) announced this fell decision, and / but immediately afterwards she reversed it.

Le modèle référentiel est ici encore $\mathrm{p} 1$ état de fait $=>$ enchaînement $=>\mathrm{p} 2$ événement ( $p 1$ state of things $=>$ sequel $=>p 2$ event). Ce que l'on remarque, c'est que $p 1$ est au preterit perfect et que la stabilisation du contenu propositionnel de p1 passe encore par l'aspect, mais par l'aspect perfect, dans lequel la stabilisation est matérialisée par have.

53 En français, on donne traditionnellement des exemples de ces deux types pour illustrer la subordination dite « subordination inverse ».

(17) Trois heures sonnaient au clocher du village quand le drame se produisit (Riegel, Pellat \& Rioul, 1998 : 507)

(18) A peine avait-il ouvert la bouche qu'on le fit taire. (Riegel, Pellat \& Rioul, 1998 : $517)^{18}$ 
serve les mêmes caractéristiques que dans les exemples déjà analysés : le contenu de 1 représente un état de fait stabilisé, et la subordination articule sur cet état de fait un développement $\mathrm{p} 2$ qui en émerge et dont le repérage temporel est fourni par p1 (trois heures sonnaient au clocher du village / A peine avait-il ouvert la bouche). L'ordre est toujours justifié par le fait que c'est le contenu préconstruit, acquis, stabilisé, p1, qui fournit le substrat, et que le développement lui est littéralement subordonné. Dans l'exemple 17, quand fonctionne de façon analogue à when dans les exemples 3 à 5. Dans l'exemple 18, la corrélation entre à peine et que rend l'ordre contraint et quand inutilisable ${ }^{19}$. Le phénomène de subordination reste le même. L'anglais (exemple 19) et le français (exemple 20) disposent d'une structure concurrente, qui intègre un comparatif temporel et son complément corrélé :

(19) No sooner had she finished that thought than the answer came straight back to her. (Cheung)

(20) Il n'avait pas plus tôt terminé cette phrase que nous eûmes les oreilles déchirées par un cri affreux qui retentissait dans le château, [...]. (Leroux)

P1 et p2 se trouvent ici encore dans un ordre contraint, pour construire un enchaînement représenté par le contenu propositionnel du complément de comparaison p2, enchaînement construit sur le soubassement de p1, contenu propositionnel stabilisé20.

\section{pertinente dans aucun des exemples observés ici.}

On peut s'interroger sur ce qui motive cette idée de "subordination inverse », mais les hypothèses ne sont pas vérifiables : $1 /$ quando est adverbe et d'emploi tardif en latin; quand a gardé son statut adverbial (dans les interrogatives, directes ou indirectes, et dans les intégratives adverbiales [ou subordonnées adverbiales, qui sont des relatives sans antécédent]) mais son aptitude à subordonner l'a fait rajouter mécaniquement au paradigme des conjonctions, ce qui ferait perdre de vue son statut adverbial (alors que les subordonnants pronoms et déterminants sont parfaitement reconnus comme pronoms subordonnants ou comme déterminants subordonnants dans la tradition linguistique francisante); 2/ à la différence de when, qui a entre autres un fonctionnement d'adverbe relatif avec antécédent, quand cède la place à où lorsqu'il a un antécédent explicite, ce qui ajoute une raison supplémentaire de perdre de vue le statut adverbial de quand, et de perdre de vue son aptitude à être adverbe subordonnant, sans antécédent explicite (mais avec antécédence temporelle, comme on l'a vu), comme dans les subordonnées temporelles examinées ici.

\section{BIBLIOGRAPHIE}

Benzitoun, C. (2013). « Description de deux fonctionnements de quand et avant que / de », in Marqueurs temporels et modaux en usage, W. De Mulder, J. Mortelmans \& T. Mortelmans (éds), Cahiers Chronos n² 26, pp. 419-435. Amsterdam : Rodopi. 
Damourette, J., Pichon, E. (1911-1940, 1970 [1936]). Des mots à la pensée. Essai de grammaire de la langue française, 7 volumes. Paris : Editions d'Artrey.

Declerck, R. (1997). When-Clauses and Temporal Structure. London: Routledge.

Guillaume, G. (1929) [1965]). Temps et verbe. Théorie des aspects, des modes et des temps. Paris : Champion.

Huddleston, R., Pullum, G.K. (2002). The Cambridge Grammar of the English Language. Cambridge: Cambridge University Press.

Joly, A., O’Kelly, D. (1990). Grammaire systématique de l'anglais. Paris : Nathan.

Kabakciev, K. (2019). 'On the History of Compositional Aspects', in Athens Journal of Philology, vol. $6, \mathrm{n}^{\circ} 3$, pp. 201-224.

Le Goffic, P. (1993a). Grammaire de la phrase française. Paris : Hachette.

Le Goffic, P. (1993b). « Les subordonnées circonstancielles et le classement formel des subordonnées », in C. Guimier (éd.), 1001 Circonstants, pp. 69-102. Caen : Presses universitaires de Caen.

Lefeuvre, F. (2015). « Analyse grammaticale et sur corpus de l'expression c'est quoi ( ?) », in G. Dostie \& P. Hadermann (éd.) La dia-variation en français actuel. Études sur corpus, approches croisées et ouvrages de référence, pp. 39-62. Coll. Sciences pour la communication. Berne : Peter Lang. https:// halshs.archives-ouvertes.fr/halshs-01519591/document

Merle, J.-M. (1997). 'Just', in C. Delmas \& A. Lancri (éds.), Langage, Langues et Linguistique, Université Paris 3, pp. 157-173. https://hal.archives-ouvertes.fr/hal-00250285v1

Merle, J.-M. (1999). « Genèse et interprétation des repères hypothétiques en milieu toncal - entre arbitraire et pertinence ", in A. Trévise (éd), LINX n41, pp. 61-74. Nanterre : Université Paris 10 Nanterre. http://hal.archives-ouvertes.fr/hal-00250282/en/

Merle, J.-M. (2001). Etude du conditionnel français et de ses traductions en anglais. Gap : Ophrys.

Merle, J.-M. (2006a). Structures et référence, https://tel.archives-ouvertes.fr/tel-00672594/ document.

Merle, J.-M. (2006b). «Wh- et la référence qualitative aux paradigmes », in J.-C. Souesme (éd), Le Qualitatif, Cycnos, vol. 23, n 1, pp. 25-43. Nice : Université Nice Sophia Antipolis. http:// revel.unice.fr/cycnos/index.html?id=294

Merle, J.-M. (2012). « La qualification, phénomène protéiforme - à propos de quelques manifestations syntaxiques de la qualification en anglais et en français », in J.-M. Merle (éd), La qualification, Travaux du CLAIX n² 22, pp. 151-184. Aix-en-Provence : PUP. http://hal.archivesouvertes.fr/hal-00671423.

Merle, J.-M. (2017). « La prédication : approche de la théorie des opérations prédicatives et énonciatives ", in L. Vincent-Durroux (éd) Prédication et prépositions en anglais : la mise en relation au prisme de différentes approches linguistiques, Corela HS-22, URL : http://journals.openedition.org/ corela/4959; DOI : 10.4000/corela.4959.

Merle, J.-M. (2019). « La question et l'interrogation en contexte : point de vue énonciatif », in Corela [En ligne], HS-29 | 2019, URL : http://journals.openedition.org/corela/8834 ; DOI : https:// doi.org/10.4000/corela.8834.

Merle, J.-M. (2020a). « Problématiques linguistiques : l'aspect en anglais », https://hal.archivesouvertes.fr/hal-02476751/document. 
Merle, J.-M. (2020b). « Participe télique, participe atélique et aspect », journée d'étude L'aspect, Bordeaux février 2020, https://hal.archives-ouvertes.fr/hal-02504852.

Merle, J.-M. (2020c, à paraître). « L'anaphore en contexte, approche énonciative », in Corela.

Merle, J.-M. (2020d, à paraître). « La pluri-modalité », actes du colloque « Le possible et le nécessaire - domaines conceptuels et expression de la modalité », en hommage à Paul Larreya, Université de Pau et des Pays de l'Adour, octobre 2019.

Miller, J., Weinert R. (1998). Spontaneous Spoken Language - Syntax and Discourse. Oxford: Oxford University Press.

Quirk, R., Greenbaum, S., Leech, G., Starvik, J., Crystal, D. (1985). A Comprehensive Grammar of the English Language. Londres, New York: Longman.

\section{NOTES}

1. Je remercie Marie Loiseau et les relecteurs anonymes pour leurs remarques et critiques.

2. Autrement dit, il existe bien une syntaxe du mensonge, et on peut faire mentir la syntaxe, mais il n'existe pas de syntaxe mensongère, simplement une approche mensongère de la syntaxe.

3. Intégratives adverbiales: cf. Damourette \& Pichon [1936] 1970; Le Goffic 1993a; Lefeuvre 2015 ; Merle 2006a \& b.

4. La terminologie a beaucoup de mal à rendre compte de l'aspect en rattachant la description de l'aspect comme celle du temps aux tiroirs du temps grammatical : le plus-que-parfait avait fini de l'exemple 1 combine «aspect accompli» (parfait avoir fini de avait fini) et «temps de l'inaccompli » selon les termes de Riegel, Pellat \& Rioul (ibidem) (imparfait 'avait' de 'avait fini'), ce qui donne un « inaccompli d'accompli » ou un « accompli inaccompli », difficile à appréhender. On a affaire à un toncal (emprunt à Damourette \& Pichon 1970 [1936] : -ait, dans avait, marque l'appartenance à une actualité toncale, actualité en rupture avec l'actualité noncale, l'actualité noncale étant celle qui englobe les coordonnées origine ; cf. aussi Merle 1999, 2001) ; ce toncal (plus que parfait) exploite l'atélicité de avait et la télicité du participe télique (fini, dans avait fini) (cf. Merle 2020a \& b) pour construire un point de vue rétrospectif; il s'agit donc d'un toncal rétrospectif (mon terme pour plus que parfait), d'un regard en arrière; Damourette et Pichon le nomment toncal antérieur); c'est cette combinaison qui donne à un événement un statut d'état de fait, d'accompli stabilisé. L'événement n'est plus envisagé comme événement, avec son intériorité et/ou ses bornes d'événement, mais comme état de fait, dans la transcendance de l'événement : le point de vue rétrospectif active la pertinence du temps d'univers et éteint celle du temps d'événement (cf. Guillaume 1929 [1965], Joly \& O'Kelly 1990, Merle 2020b). Et c'est cette stabilité qui donne un caractère préconstruit à p1, et qui permet l'emploi du plus-que-parfait tout autant que de l'imparfait dans la principale des constructions qui nous intéressent ici.

5. Dans tout cet article, p1 renvoie au contenu propositionnel de la principale, p2 au contenu propositionnel de la subordonnée, quel que soit l'ordre des deux propositions.

6. Cf. Merle $2006 \mathrm{~b}$.

7. Les adverbes intégratifs (when, dans when I opened the door $=>$ the moment when) partagent avec les pronoms intégratifs (whoever dans whoever did the job $\Rightarrow>$ the person who) et avec les déterminants intégratifs (what dans what money she had => the money that she had) la propriété de ne pas avoir d'antécédence.

Référentiellement, la première glose n'est qu'une étape approximative, puisqu'elle reconstruit une référence à du non quelconque non spécifié, l'antécédence étant une forme de spécification. Or ce non quelconque, dans les intégratives adverbiales à référence spécifique comme celles des 
exemples 6 à 8, est la coordonnée temporelle de p2. Une fois qu'elle est matérialisée par p2, cette coordonnée temporelle est non quelconque et spécifiée.

8. Cf. Le Goffic 1993b. On peut faire la même remarque à propos des intégratives nominales qu'à propos des intégratives adverbiales : les intégratives adverbiales ont une fonction adverbiale et leur subordonnant a lui-même une fonction adverbiale à l'intérieur de l'intégrative adverbiale (whenever she came, she felt at ease) ; les intégratives nominales ont une fonction nominale et leur subordonnant a lui-même une fonction nominale à l'intérieur de l'intégrative nominale (whoever did the job did an excellent job).

9. N'ayant pas d'antécédent nominal, elles ne peuvent être intégrées dans un syntagme nominal et ne peuvent avoir une fonction épithétique; leur fonction est fondamentalement modale et déborde très largement le cadre de la modalité appréciative.

10. Ce qui ne fait pas du relatif un coordonnant.

11. Ce qui permet de faire exister et d'exploiter une structure qui a des caractéristiques symétriques de celles des structures extraposées (cf. He wouldn't let us defend ourselves, which was completely unfair. VS It was completely unfair that he wouldn't let us defend ourselves). Seul le contexte permettrait d'opposer les motivations (ou le « fait essentiel») de chacune des deux structures ; la première, co-prédicative, repose sur une co-assertion, le contenu de la relative appositive étant essentiellement appréciatif (co-assertion appréciative) ; la seconde est l'assertion d'un jugement (ici, jugement appréciatif) sur le contenu propositionnel extraposé. Les deux sont pluri-modales.

12. Cf. Merle 2017 ou 2020d, à paraître.

13. Proform est un terme mis en circulation par Quirk \& al. 1985. L'idée de départ est que pro-nom «mis pour un nom ", littéralement - devrait se comprendre par rapport à un antécédent (définition référentielle, non-viable). Si un pronom, selon cette approche, a un antécédent qui est un syntagme nominal, ou une proposition, ou un énoncé, on ne doit pas l'appeler 'pro-nom', dans cet esprit, mais 'pro-syntagme nominal', 'pro-proposition', 'pro-énoncé', etc. D’où le terme de proforme, qui donne l'idée qu'on a affaire à pro-autre-que-nom, et qui entretient l'idée que le terme de pronom s'applique aux cas particuliers dont l'antécédent est un nom propre ou un nom à déterminant $\emptyset$. Comme cette définition s'appuie à la fois sur des critères morphologiques et référentiels, elle a un grand pouvoir de séduction, mais elle n'est pas viable: 1 / une catégorie syntaxique a besoin d'une définition syntaxique, et la catégorie du pronom est une «catégorie grammaticale à fonction nominale » (cf. Merle 2020c, à paraitre) ; 2/ l'idée d'une classe de proformes définies comme renvoyant à "un-antécédent-autre-que-nom » exclut ipso facto de la classe des pronoms et des proformes tous les pronoms qui n'ont pas d'antécédent: $2 \mathrm{a} /$ les pronoms déictiques ; $2 \mathrm{~b}$ / les pronoms paradigmatiques (indéfinis) ; $2 \mathrm{c} /$ les pronoms en $w h-/ q u-$ quand ils sont sans antécédent; etc.

Les pronoms sont en fait une catégorie grammaticale à fonction nominale et le pendant grammatical de la classe lexicale du nom.

Mais le terme de proforme est une création particulièrement utile, qui peut s'employer pour toutes les formes (simples ou complexes) qui n'ont pas de fonction nominale, mais un fonctionnement comparable à celui des pronoms, notamment dans le paradigme des prédicats, dans celui des représentations d'états de fait et dans celui des représentations d'événements.

1 / Proformes simples - Ex. I don't think she will help much but she might $\underline{\text { do. }}$.

2/ Proformes complexes

2a/ Proformes déictiques do this / be this / this happened / fais ceci / etc.

$2 b$ / proformes anaphoriques do that / do so / do it / be that / fais-le / it happened / etc.

2c/ Proformes paradigmatiques do something / do nothing / faire quelque chose / faire quoi ? do what? / be what? / nothing happened / etc.

On a beaucoup à y gagner: on a tendance à ne reconnaître que ce que l'on nomme, et inversement; en donnant un nom et une définition aux proformes, on peut alors en reconnaître l'existence, et les étudier. 
14. Référentiellement, les prédicats renvoient à des procès (statiques comme dans l'exemple 9 , ou dynamiques comme dans l'exemple 10), les syntagmes nominaux à des référents, et les relations prédicatives à des états de fait ou à des événements.

15. Propositions relatives, syntagmes participiaux, syntagmes prépositionnels, syntagmes adjectivaux, noms (cf. Merle 2012).

16. Ex. I am a poor lonesome cowboy.

17. L'opposition entre relative intégrée et relative appositive recouvre tous les cas de figure même si elle inclut des ambiguïtés, alors que l'opposition entre relatives appositives et relatives déterminatives n'est pas plus viable, mutatis mutandis, qu'une opposition, dans le domaine des moyens de transport, entre vélos, d'un côté, et voitures rouges, de l'autre : les voitures rouges sont un cas particulier de voiture; de même les relatives déterminatives sont un cas particulier d'emploi (à fonction référentielle) des relatives intégrées. Les relatives intégrées sont intégrées pour représenter une propriété saillante, caractéristique, inhérente à leur support-antécédent.

18. Riegel, Pellat \& Rioul (1998: 517) font un commentaire analogue à celui qui accompagne l'exemple 17 (citation $1, \S 1$ ) : «La première proposition est ou de type négatif (elle contient ne pas, ne pas encore, ne pas plutôt); ou au moins d'orientation argumentative négative avec à peine. Mais c'est en réalité l'expression d'une circonstance. La seconde, qui contient le fait principal, est introduite par que : il s'agit donc d'un cas de subordination inverse [...]. »

Cf. $\$ 1$ ci-dessus, remarque 1. L' " orientation argumentative négative » de à peine est en fait temporelle (cf. Merle 1997)

19. L'emploi de quand aurait pour effet ici de générer une intégrative adverbiale.

20. La stabilisation est opérée par l'aspect : dans l'exemple 20, l'aspect pourrait être porté par un "passé antérieur» - il n'eut pas plus tôt [...] - l'antériorité marquée par l'aspect (reflet dans l'énoncé d'un point de vue rétrospectif) et la négation d'antériorité coexistant non pour se contredire, mais pour focaliser sur l'immédiateté de l'enchaînement.

\section{RÉSUMÉS}

Cet article revient sur un phénomène syntaxique, en anglais et en français - la subordination temporelle dite « subordination inverse » (Il avait fini son travail sur les ruches [...] quand il aperçut David Kellerman qui s'activait [Teissier] /// He was just congratulating himself on his keen sense of observation [...], when the truck smashed into his car [...] [Bradbury].). On définit traditionnellement ce phénomène $(\$ 1)$ à partir de gloses qui remanient la syntaxe (Alors qu'il avait fini son travail [...] il aperçut David Kellerman / As he was congratulating himself [...] the truck smashed into his car). L'observation passe par trois étapes successives. Comme le phénomène de « subordination inverse » concerne les subordonnées temporelles, la première étape (§ 2) est un retour sur le repérage temporel opéré par les subordonnées intégratives adverbiales. La deuxième étape (\$ 3 ) revient sur les caractéristiques des relatives à antécédent non nominal. La troisième étape $(\S 4)$ est consacrée aux relatives continuatives. Le $\S 5$ fait la synthèse des observations : cette subordination temporelle n'est pas «subordination inverse »; comme le fait toujours la syntaxe, elle apporte ici sa contribution à la cohérence discursive et à la construction du sens.

This paper is about so-called reverse subordination in French and in English (Il avait fini son travail sur les ruches [...] quand il aperçut David Kellerman qui s'activait [Teissier] /// He was 
just congratulating himself on his keen sense of observation [...], when the truck smashed into his car [...] [Bradbury].). The idea of 'reverse subordination' is traditionally based on paraphrases reshuffling the syntax (§ 1) (Alors qu'il avait fini son travail [...] il aperçut David Kellerman / As he was congratulating himself [...] the truck smashed into his car). Observation follows three successive stages. As 'reverse subordination' concerns temporal subclauses, the first stage (\$2) is the location in time operated by temporal adverbial clauses. The second stage ( $\$ 3)$ revisits relative clauses with non-nominal antecedents. The third stage (\$4) examines continuative relative clauses. $\S 5$ is a synthesis of observations: syntax as always contributes to discursive cohesion and the construction of meaning, and the temporal subordination under study need not be reanalyzed as 'reverse subordination'.

\section{INDEX}

Mots-clés : subordination inverse ; gloses ; subordonnées temporelles ; relatives à antécédent non nominal ; relatives continuatives ; cohérence discursive

Keywords : Reverse subordination; paraphrases; temporal subordinate clauses; relative clauses with non-nominal antecedents; continuative relative clauses; discursive cohesion

\section{AUTEUR}

\section{JEAN-MARIE MERLE}

Université Nice Sophia Antipolis 